\title{
Metabolic Effects of Valproic Acid Augmentation in Male Schizophrenic Patients
}

\author{
Takahiko Nagamine, M.D., Ph.D. \\ Division of Psychiatric Internal Medicine, \\ Seiwakai-Kitsunan Hospital
}

Received September 18, 2012 / Accepted September 26, 2012 / Published November 20, 2012

Atypical antipsychotics are associated with a risk of metabolic disorders such as diabetes mellitus and hyperlipidemia [1]. Valproic acid may produce different metabolic effects when combined with various atypical antipsychotic drugs [2]. Weight gain and/or unfavorable lipid changes during valproate treatment may be observed within the first 3 months of therapy and women seem to be more susceptible than men [3]. Longitudinal metabolic data of valproic acid augmentation are lacking in male psychotic patients.

A retrospective study was conducted on the metabolic risk in male patients with DSM-IV-TR schizophrenia in whom treatment was initiated with risperidone, olanzapine or blonanserin and treatment with valproic acid was added 3-12 weeks after treatment initiation. Body weight and non-high-density lipoprotein cholesterol (non-HDL-C) levels in individual patients were measured at 3 time points: before antipsychotic drug treatment initiation; immediately before the valproic acid treatment initiation (within 1 week before initiation) and 10-12 weeks after valproic acid add-on treatment initiation. Non-HDL-C, calculated by subtracting HDL-C from total cholesterol, is advantageous in that it correlates with triglyceride and is unlikely to be affected by diet. Assessment of non-HDL-C provides a measure of cholesterol contained in all atherogenic particles [4]. Patients having evident metabolic disorders (e.g., diabetes mellitus) and patients receiving drug therapy for diabetes mellitus or hyperlipidemia were excluded from the study. Three points (before treatment, antipsychotic monotherapy, valproic acid add-on therapy) were compared using repeated measures ANOVA for each antipsychotic, with $\mathrm{p}<0.05$ indicating a significant difference. The values were expressed as the mean \pm standard deviation.

The analysis set consisted of 26 patients in total, with the initial drug being risperidone in 11 patients (the risperidone group), olanzapine in 8 patients (the olanzapine group) and blonanserin in 7 patients (the blonanserin group). The length of time from the start of antipsychotic drug treatment to the addition of valproic acid was $4.6 \pm 2.8$ weeks in the risperidone group, $4.4 \pm 3.0$ weeks in the olanzapine group, and $4.0 \pm 2.9$ weeks in the blonanserin group. Thus, valproic acid began to be used within 6 weeks after treatment initiation in more than half of all patients. The age and the dose level of the antipsychotic drug and valproic acid in each group were as follows. In the risperidone group, the age was $42.3 \pm 12.9$ years, the risperidone dose was $5.9 \pm 3.4 \mathrm{mg}$ and the valproic acid dose was $761 \pm 375 \mathrm{mg}$. In the olanzapine group, the age was $38.3 \pm 13.3$ years, the olanzapine dose was $16.5 \pm 5.3 \mathrm{mg}$ and the valproic acid dose was 744 $\pm 359 \mathrm{mg}$. In the blonanserin group, the age was $40.8 \pm 16.1$ years, the blonanserin dose was $14.2 \pm 9.8$ $\mathrm{mg}$ and the valproic acid dose was $804 \pm 304 \mathrm{mg}$.

Figure 1 shows the change over time in the mean body weight in each group and Figure 2 shows the change over time in the mean non-HDL-C level. Weight gain during the antipsychotic monotherapy and during the valproic acid add-on therapy was $1.9 \pm$ $0.8 \mathrm{~kg}$ and $2.2 \pm 1.1 \mathrm{~kg}$ in the risperidone group, $7.0 \pm$ $3.1 \mathrm{~kg}$ and $1.8 \pm 0.8 \mathrm{~kg}$ in the olanzapine group and $0.6 \pm 0.5 \mathrm{~kg}$ and $1.2 \pm 0.9 \mathrm{~kg}$ in the blonanserin group, respectively. The magnitude of the increase in the non-HDL-C level during the antipsychotic monotherapy and during the valproic acid add-on 
therapy was $9.8 \pm 6.2 \mathrm{mg} / \mathrm{dL}$ and $12.4 \pm 4.3 \mathrm{mg} / \mathrm{dL}$ in the risperidone group, $26.0 \pm 7.3 \mathrm{mg} / \mathrm{dL}$ and $16.4 \pm$ $6.4 \mathrm{mg} / \mathrm{dL}$ in the olanzapine group and $-1.0 \pm$ $1.8 \mathrm{mg} / \mathrm{dL}$ and $2.3 \pm 2.1 \mathrm{mg} / \mathrm{dL}$ in the blonanserin group, respectively.

In the risperidone group, significant weight gain was noted during the add-on valproic acid treatment period compared before treatment initiation. In the olanzapine group, significant weight gain was noted during both the antipsychotic monotherapy period and the valproic acid add-on treatment period compared to before treatment initiation. The blonanserin group exhibited no significant weight gain. The non-HDL-C level increased significantly during the valproic acid add-on treatment period compared to before treatment initiation in the risperidone group. In the olanzapine group, the non-HDL-C level increased significantly during the antipsychotic monotherapy period and the valproic acid add-on treatment period compared to before treatment initiation, and the level was signifi- cantly higher during the valproic acid add-on treatment period than during the antipsychotic monotherapy period. No significant increase in the non-HDL-C level was noted in the blonanserin group. Valproic acid augmentation was associated with higher body weight as well as elevated non-HDL-C levels in the olanzapine and risperidone groups but not in the blonanserin group. These results indicate that both some atypical antipsychotic drugs and valproic acid are associated with a risk of metabolic disorders. It seems desirable to analyze the risk stemming from the combined use of these drugs because the mechanism through which valproic acid may induce weight gain is still debated. The limitations of the present study are that it was retrospectively conducted and the sample size was very small. The association between valproic acid add-on therapy and metabolic abnormalities, as evidenced by, for example, measurement of fasting blood glucose, insulin level and $\mathrm{HbAlc}$, needs to be studied prospectively.

Figure 1. Body weight changes after antipsychotic and valproic acid add-on therapy

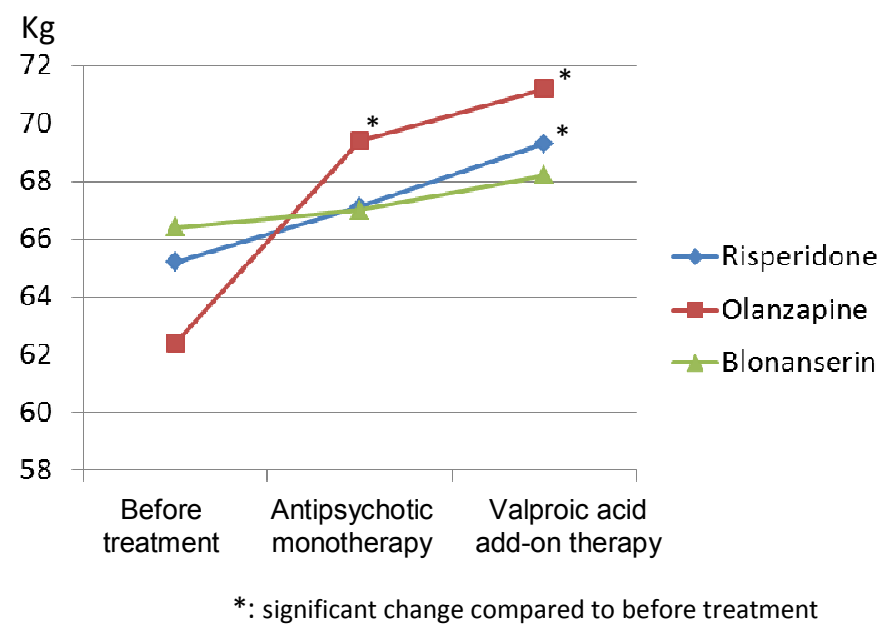

Figure 2. Non-HDL cholesterol changes after antipsychotic and valproic acid add-on therapy

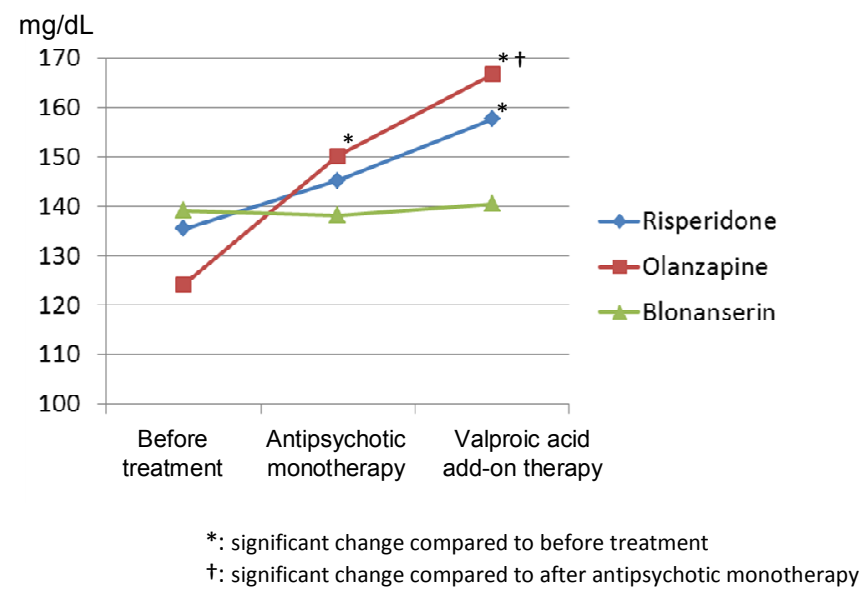




\section{DISCLOSURE}

The author reports no conflicts of interest in this research.

\section{REFERENCES}

[1] Nagamine T. Effects of risperidone and olanzapine on remnant-like lipoprotein particle cholesterol (RLP-C) in schizophrenic patients. Neuropsychiatr Dis Treat. 2008;4(2):481-6.

[2] Meltzer HY, Bonaccorso S, Bobo WV, Chen Y, Jayathilake K. A 12-month randomized, openlabel study of the metabolic effects of olanzapine and risperidone in psychotic patients: influence of valproic acid augmentation. J Clin Psychiatry. 2011;72(12):1602-10.

[3] Verrotti A, D'Egidio C, Mohn A, Coppola G, Chiarelli F. Weight gain following treatment with valproic acid: pathogenetic mechanisms and clinical implications. Obes Rev. 2011;12(5): e32-43.

[4] Rana JS, Boekholdt SM, Kastelein JJ, Shah PK. The role of non-HDL cholesterol in risk stratification for coronary artery disease. Curr Atheroscler Rep. 2012 ;14(2):130-4. 